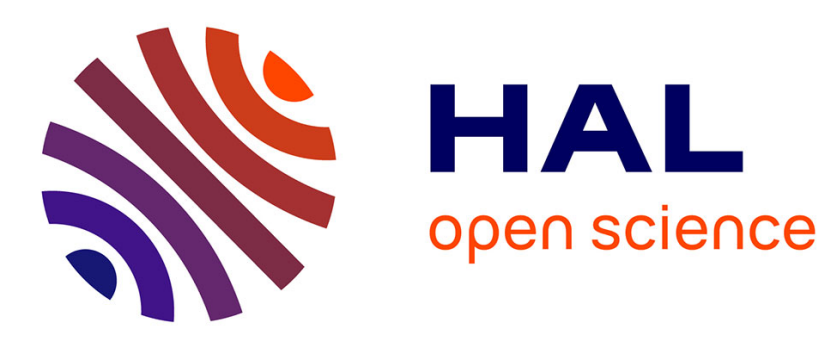

\title{
Three-dimensional face recognition using elastic deformations of facial surfaces
}

Mohamed Daoudi, Lahoucine Ballihi, Chafik Samir, Anuj Srivastava

\section{To cite this version:}

Mohamed Daoudi, Lahoucine Ballihi, Chafik Samir, Anuj Srivastava. Three-dimensional face recognition using elastic deformations of facial surfaces. 2008 IEEE International Conference on Multimedia \& Expo, Jun 2008, Hannover, Germany. pp.97-100. hal-00665585

\section{HAL Id: hal-00665585 https://hal.science/hal-00665585}

Submitted on 3 Feb 2012

HAL is a multi-disciplinary open access archive for the deposit and dissemination of scientific research documents, whether they are published or not. The documents may come from teaching and research institutions in France or abroad, or from public or private research centers.
L'archive ouverte pluridisciplinaire HAL, est destinée au dépôt et à la diffusion de documents scientifiques de niveau recherche, publiés ou non, émanant des établissements d'enseignement et de recherche français ou étrangers, des laboratoires publics ou privés. 


\section{THREE-DIMENSIONAL FACE RECOGNITION USING ELASTIC DEFORMATIONS OF FACIAL SURFACES}

\author{
Mohamed Daoudi ${ }^{1}$, Lahoucine Ballihi ${ }^{1,2}$, Chafik Samir ${ }^{1}$ \\ ${ }^{1}$ Institut TELECOM ; TELECOM Lille1 ; CNRS LIFL \\ ${ }^{2}$ GSCM/LRIT, Faculté des Sciences, Rabat, Morocco
}

\author{
Anuj Srivastava
}

Department of Statistics
Florida State University
Tallahassee, FL 32306

\begin{abstract}
We propose a pattern theoretic approach for studying variability in shapes of facial surfaces. Our idea is to impose a specific, yet natural, coordinate system, called a curvilinear coordinate system, on facial surfaces. In this system, one coordinate $\xi_{1}$ measures the distance of a point from the tip of the nose and its level curves are called the facial curves. The other coordinate $\xi_{2}$ measures distances along these curves; level curves of this coordinate are orthogonal to the facial curves. To compare two facial surfaces we use elastic deformations that use stretching, shrinking, and bending to optimally register points across two surfaces. We will demonstrate this idea on Florida State University (FSU) 3D face database.
\end{abstract}

Index Terms - 3D face recognition, curvilinear coordinate system, elastic deformation.

\section{INTRODUCTION}

Automatic face recognition has actively been researched in recent years, and various techniques using ideas from $2 \mathrm{D}$ image analysis have been presented. Although a significant progress has been made, the task of automated, robust face recognition is still a distant goal. 2D Image-based methods are inherently limited by variability in imaging factors such as illumination and pose.

An emerging solution is to use 3D imaging sensors for capturing surfaces of human faces, and use these data in performing face recognition. Such observations are relatively invariant to illumination and pose, although they do vary with facial expressions. As the technology for acquiring 3D facial surfaces becomes simpler and cheaper, the use of 3D facial scans will be increasingly prominent. Trying to use 3D information has become an emerging research direction in hope to make face recognition more accurate and robust.

A first approach proposes to apply classical 2D methods for $3 \mathrm{D}$ face recognition by considering range (depth) images. Hesher et al. [1] presented an efficient technique to compare certain aspects of facial shapes using ideas from image analysis: a search for optimal subspace based on stochastic gradient algorithm on a Grassman manifold was proposed.
The second approach proposes to represent facial surfaces by certain geometrical features sets, such as the convex parts, areas with high curvatures, saddle points, etc [2]. For surface matching, the authors used classical 3D surface alignment algorithms, typically ICP (Iterative Closest Point), that computes the residual error between the probe surface and the $3 \mathrm{D}$ images in the gallery. ICP is a procedure which iteratively minimizes the distance between two sets of points. Only a rigid transformation is taken into account in this type of algorithms. Although such feature definitions are intuitively meaningful, the computation of curvatures involves numerical approximation of second derivatives, and thus is very susceptible to observation noise. In [3] and [4], facial surfaces have been approximated with indexed sets of level curves of a continuous function, such as the depth function, defined on these surfaces. Level curves of depth function are extracted efficiently using standard techniques on range images. Since these curves are planar, the authors used shape analysis of planar curves [5] to comparing and deforming faces; matching problem was not studied here (that is, the mapping was linear). The metric used was the non-elastic metric with the method used for computing geodesics being the shooting method. This representation was not fully invariant to rigid motions of the face. All these approaches treat the face as a rigid object and do not perform well in the presence of expression variation. However, an important requirement of a face analysis system is the non-rigid of points across faces.

Bronstein et al. [6] use a geodesic distance function to define level curves that are invariant to rigid motions and also to facial expressions to some extent. The authors present a new proposal which considers the facial surface (only a frontal view) as an isometric surface (length preserving). Using a global transformation based on geodesics, they obtain invariant forms to facial expressions. After the transformation, they perform one classical rigid surface matching and PCA for sub-space building and face matching. The authors resolve partially the problem of facial expression variation. The results shown were obtained on a small data set containing 220 faces of 30 subjects - 3 artificial and 27 human. Chang et al. 


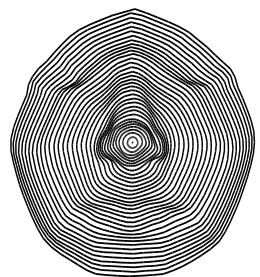

(a)

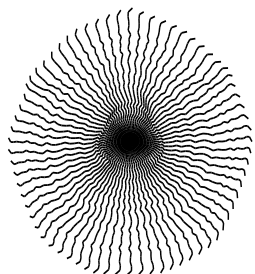

(b)

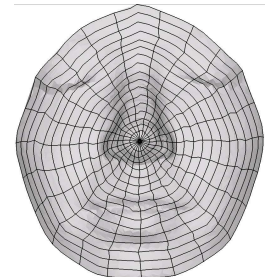

(c)
Fig. 1. (a) Level curves of $\xi_{1}$, (b) level curves of $\xi_{2}$ for three different facial surfaces, (c) full curvilinear coordinate system on some face.

[7], explore an approach that matches multiple, overlapping surface patches around the nose area and then combines the results from these matches to achieve greater accuracy. This work seeks to explore what can be achieved by using a subset of the face surface that is approximately rigid across expression variation. Kakadiaris et al. [8] utilize an annotated face model to study geometrical variability across faces.

In this paper we present a unified Riemannian framework that provides optimal matching, comparisons and deformations of faces using a single elastic metric based on curvilinear coordinate system. We propose, the construction of geodesic paths between curvilinear coordinate system curves by using an elastic metric which allows us to realize "optimal" deformations between surfaces.

The rest of this paper is organized as follows: Section 2 describes the representation of a facial surface using a curvilinear coordinate system, and presents a metric for comparing facial shapes under this representation. Section 3 presents experimental results using FSU database. We finish the paper with a brief summary in Section 4 .

\section{ELASTIC DISTANCES BETWEEN TWO FACIAL SURFACES}

The starting point of our framework is to define an intrinsic coordinate system on each facial surface that is natural for studying shape variability. This system is used to decompose elastic matching/deformation of facial surfaces into simpler, more elementary components.

\subsection{Curvilinear Coordinate System}

Let $S$ be a facial surface assumed to be a two-dimensional differentiable manifold that contains its boundaries. This is obtained in practice by filling the holes in the data obtained from 3D scanners, and by smoothly interpolating the resulting mesh to ensure two-dimensionality of any patch on the surface. Furthermore, $S$ is endowed with a Riemannian structure using the Euclidean metric in $\mathbb{R}^{2}$, the tangent space at any interior point of $S$. Let $r \in S$ denote the tip of the nose on
$S$. For any point $p \in S$, define a function $f: S \mapsto \mathbb{R}$ such that $f(p)$ is the length of the shortest geodesic from $r$ to $p$. Since there exists a shortest geodesic from $r$ to $p$, for all $p$, the function $f$ is well defined. Also, since $S$ is a differentiable manifold, the function $f$ is a continuous function.

We define a coordinate system on $S$ using the function $f$ as follows. We define the level curves of $f$ as the level curves of the first coordinate $\xi_{1}$, the value of $\xi_{1}$ being equal to the value of $f$. Shown in the Figure 1 is an example of these curves on a face. Next, we use the streamlines of $\nabla f$, starting from $r$, as the level curves of the second coordinate $\xi_{2}$. The value of $\xi_{2}$ along such curves will be dependent on the initial directions of these streamlines. Together, these two coordinates provide a well-defined, orthogonal coordinate system.

\subsection{Elastic Deformation of Facial Surfaces}

Once the curvilinear coordinate system is established, the task of elastically matching two surfaces is that of finding a mapping $\phi: S_{1} \mapsto S_{2}$ that is optimal under the elastic metric. In our approach, $\phi$ can be decomposed into simpler components. There are two ways of decomposing $\phi\left(\xi_{1}, \xi_{2}\right)$ : (i) into $\phi_{1}\left(\xi_{1}\right) \phi_{2}\left(\xi_{2} ; \xi_{1}\right)$ and (ii) into $\phi_{2}\left(\xi_{2}\right) \phi_{1}\left(\xi_{1} ; \xi_{2}\right)$. In the former decomposition, we first match the level curves of $\xi_{1}$, to find $\phi_{1}$, and then for each pair of matched curves we find $\phi_{2}$.

In [9] we have proposed a solution to estimate $\phi^{*}\left(\xi_{1}, \xi_{2}\right)=$ $\phi_{1}^{*}\left(\xi_{1}\right) \phi_{2}^{*}\left(\xi_{2} ; \xi_{1}\right)$. Firstly, to estimate $\phi_{2}^{*}$, we propose to use the approach proposed by Mio et al. [10]. The main steps of this approach are (i) defining a space of closed curves, (ii) imposing a Riemannian metric structure on this space using the elastic metric, and (iii) computing geodesic paths under this path. This approach provides a solution for solving optimal mapping $\phi_{2}^{*}$. Secondly, to estimate $\phi_{1}^{*}$, we have proposes to use a dynamic programming [9]; please refer to that paper for details. In this paper we assume that $\phi_{1}^{*}=I d$.

The construction of geodesic paths between curvilinear coordinates curves by using an elastic metric proposed by Mio et al. [10], allows us to realize optimal deformations between surfaces. Applying this idea repeatedly to each pair of registered curves gives us a full elastic deformation from one face to another. If we view each facial surface as an indexed collection of curves, indexed by $\xi_{1}$, this idea provides a path on the space of such collections, in going from one face to another. We will denote this path by $\Omega$, where $\Omega(t)$, for any $t \in[0,1]$ is an indexed collection of points in $\mathcal{S}$. This path is optimal in the sense that it minimizes the total length:

$$
d\left(S_{1}, S_{2}\right) \equiv \int_{0}^{L} d_{s}\left(C_{\xi_{1}}^{1}, C_{\phi_{1}^{*}\left(\xi_{1}\right)}^{2}\right) d \xi_{1} .
$$

where

$C_{\xi_{1}}^{1}$ and $C_{\xi_{1}^{\prime}}^{2}$ are two arbitrary level curves of the first coordinate in facial surfaces $S_{1}$ and $S_{2}$, respectively, and $d_{s}$ is the geodesic distance two curves defined in [9]. 
Figure 2 shows two examples of deforming facial surfaces, one into another, when they both faces belong to the same person (top) and when the faces belong to different persons (bottom).

\section{EXPERIMENTAL RESULTS}

In this section we present some experimental results to demonstrate effectiveness of our approach. We setup recognition experiments where we use a fraction of faces as gallery and the remaining as probe and use the distance $d\left(S_{1}, S_{2}\right)$ (Eqn. 1) and the nearest neighbor classifier to classify the probe faces.

We performed a series of experiments using FSU database to assess the performance of our algorithm and to evaluate its robustness to facial expressions. We used a total of 192 facial surfaces. The data were acquired using a Minolta Vivid700 laser scanner that produces 3D faces. We divide this set into gallery which consists of a total of 96 neutral facial surfaces and probe sets which consists of a total of 96 non neutral expression (neutral, smile, frown, angry, squint, scared) taking randomly.

The results are summarized using Cross Correlation Matrix and Receiver Operating Characteristic (ROC) curve. The Cross Correlation Matrix is shown as a matrix in Figure 3. Experiments show that the proposed elastic geodesic distance is stable to changes in facial expressions. That is, the deformation caused by changes in expressions is much smaller in terms of the geodesic distances, when compared to the distances between faces of different people except for some faces. The matrix shows that there are 12 not recognized correctly by our approach. Figure 4 shows the verification rate results of the proposed algorithm. In this case, the verification rate at $8 \%$ is $92 \%$ for probe with a neutral expression.

Figure 5 shows 4 example probes and their corresponding gallery faces, which could not be recognized by our algorithm. Figure 5 shows that the tip of the nose detection algorithm fails to detect correctly a tip of the nose of the face in the top. Indeed, the curvilinear coordinate system relies critically on the location of the reference point (tip of the nose), and there is a possibility of numerical error in estimating this point.

\section{SUMMARY}

We have described an elastic deformations algorithms for comparing the shapes of facial surfaces. The first facial recognition results obtained on FSU database show the efficiency of our approach.

In the future work, we will improve the nose detection and the facial curves extraction algorithms. We will also apply our approach on FRGC V2.0 and we will compare it with other approaches.

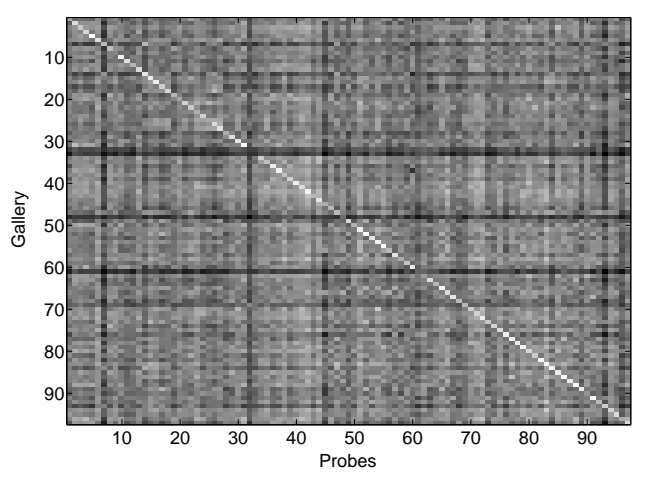

Fig. 3. Cross Correlation Matrix of the elastic algorithm.

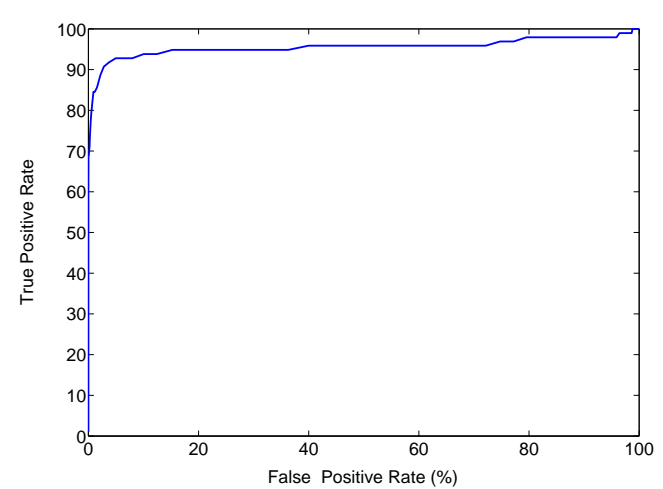

Fig. 4. ROC curve of the elastic algorithm.

\section{ACKNOWLEDGMENT}

Portions of this research were supported by the project FAR3D ANR-07-SESU-004, by the Centre National de la Recherche Scientifique, and by the Institut TELECOM under the project RecoVis3D.

\section{REFERENCES}

[1] C. Hesher, A. Srivastava, and G. Erlebacher, "A novel technique for recognizing faces using range images," in Proceedings of ISSPA, 2003, Paris, France, 2003.

[2] X. Lu, A. K. Jain, and D. Colbry, "Matching 2.5d face scans to 3d models," IEEE Transactions on Pattern Analysis and Machine Intelligence, vol. 28, no. 1, pp. 31-43, Jan. 2006.

[3] C. Samir, A. Srivastava, and M. Daoudi, "3d face recognition using shapes of facial curves," in Proceedings of ICASSP, 2006, Special Session on Statistical inferences on nonlinear manifolds with applications in signal and image processing, May, 2006. 

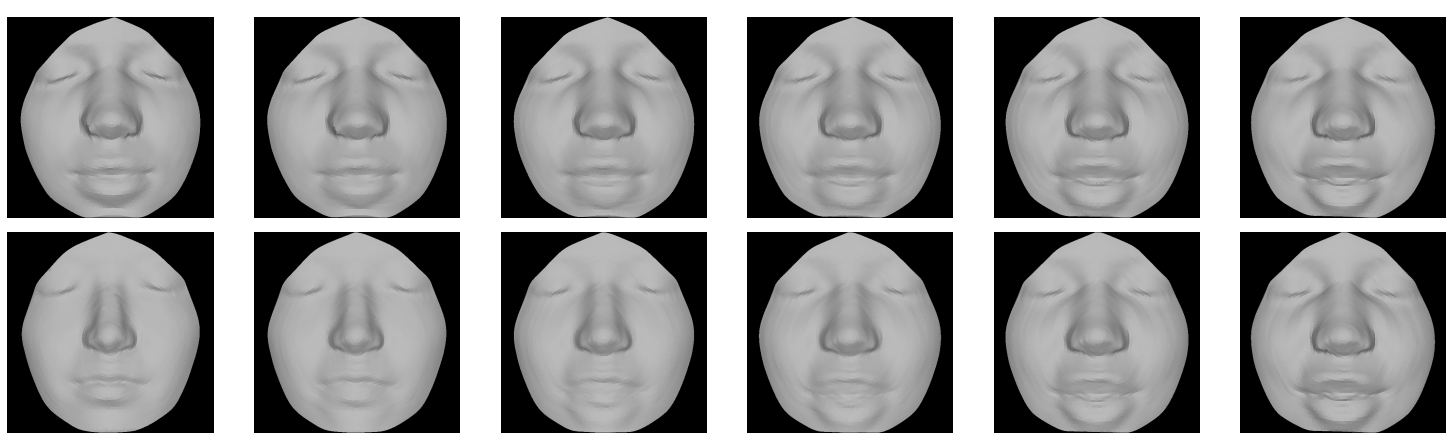

Fig. 2. Optimal deformations of facial surfaces into each other, both belonging to the same person (top), belonging to the different person (bottom) under the elastic energy.

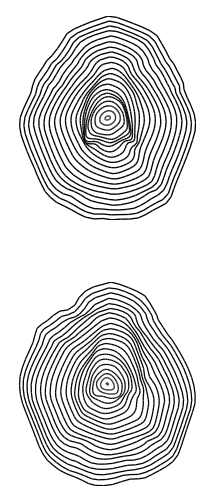

(a)
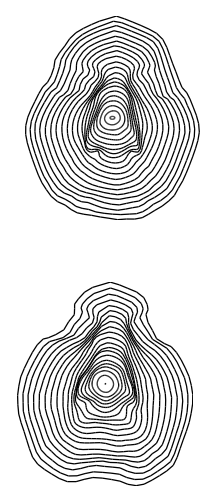

(b)
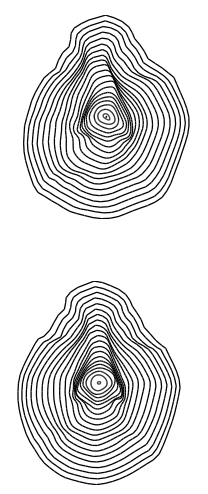

(c)
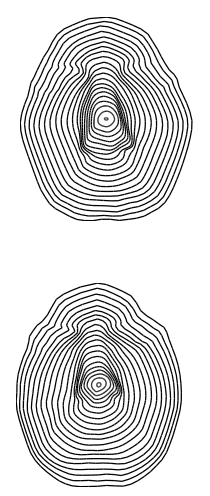

(d)

Fig. 5. Example of probe faces that could not recognized correctly (top) and their corresponding gallery faces (bottom).

[4] C. Samir, A. Srivastava, and M. Daoudi, "Threedimensional face recognition using shapes of facial curves," IEEE Transactions on Pattern Analysis and Pattern Recognition, vol. 28, no. 11, pp. 1858-1863, 2006.

[5] E. Klassen, A. Srivastava, W. Mio, and S. H. Joshi, "Analysis of planar shapes using geodesic paths on shape spaces," IEEE Trans. Pattern Analysis and Machine Intelligence, vol. 26, no. 3, pp. 372-383, 2004.

[6] A. M. Bronstein, M.M. Bronstein, and R. Kimmel, "Three-dimensional face recognition," International Journal of Computer Vision, vol. 64, no. 1, pp. 5-30, 2005.

[7] K. I. Chang, K. W. Bowyer, and P. J. Flynn, "Multiple nose region matching for 3D face recognition under varying facial expression," IEEE Transactions on Pattern Analysis and Machine Intelligence, vol. 28, no. 10, pp. 1695-1700, 2006.

[8] I. A. Kakadiaris, G. Passalis, G. Toderici, M. N. Mur- tuza, N. Karampatziakis, and T. Theoharis, "Threedimensional face recognition in the presence of facial expressions: An annotated deformable model approach," IEEE Transactions on Pattern Analysis and Machine Intelligence, vol. 29, no. 4, pp. 1-10, 2007.

[9] A. Srivastava, C. Samir, S.H. Joshi, and M. Daoudi, "Elastic shape models for face analysis using curvilinear coordinates," Journal of Mathematical Imaging and Vision, accepted.

[10] W. Mio, A. Srivastava, and S. H. Joshi, "On shape of plane elastic curves," International Journal of Computer Vision, vol. 73, no. 3, pp. 307-324, 2007. 